\title{
Simultaneously Exploiting Two Formulations: An Exact Benders Decomposition Approach
}

\author{
Lusby, Richard Martin ; Røpke, Stefan; Gamst, Mette; Spoorendonk, Simon
}

Publication date:

2018

Document Version

Peer reviewed version

Link back to DTU Orbit

Citation (APA):

Lusby, R. M. (Author), Røpke, S. (Author), Gamst, M. (Author), \& Spoorendonk, S. (Author). (2018).

Simultaneously Exploiting Two Formulations: An Exact Benders Decomposition Approach. Sound/Visual production (digital)

\section{General rights}

Copyright and moral rights for the publications made accessible in the public portal are retained by the authors and/or other copyright owners and it is a condition of accessing publications that users recognise and abide by the legal requirements associated with these rights.

- Users may download and print one copy of any publication from the public portal for the purpose of private study or research.

- You may not further distribute the material or use it for any profit-making activity or commercial gain

- You may freely distribute the URL identifying the publication in the public portal

If you believe that this document breaches copyright please contact us providing details, and we will remove access to the work immediately and investigate your claim. 


\section{Simultaneously Exploiting Two Formulations:}

\section{An Exact Benders Decomposition Approach}

Richard M. Lusby Stefan Røpke Mette Gamst Simon Spoorendonk

DTU Management Enginnering

The Technical University of Denmark

EURO 2018

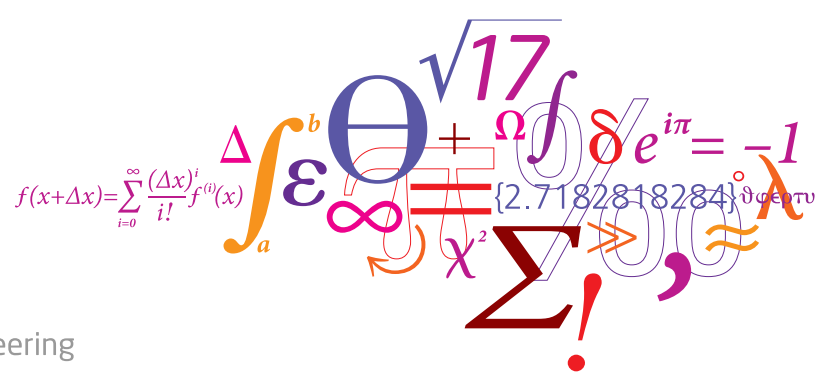

DTU Management Engineering

Department of Management Engineering 


\section{Agenda}

(1) Combining Two MIP Formulations

- Reformulations and Relaxations

- Benders Decomposition

2 Application One

- The Bin Packing Problem

(3) Application Two

- The Split Delivery Vehicle Routing Problem (SDVRP)

(4) Summary 


\section{Motivation}

- Multiple ways to model a problem using mixed integer linear programming

- Each formulation may have its own advantages and disadvantages

$\rightarrow$ Branching in one is easier than the other

$\rightarrow$ One provides a tighter relaxation than the other

- Can we couple two separate formulations together to exploit the benefits of each?

- Solve the resulting formulation using Benders Decomposition

- We consider two different cases:

$\rightarrow$ One formulation is a reformulation of the other

$\rightarrow$ One formulation is a relaxation of the other 
Methodology (1)

$$
\begin{aligned}
& (\mathcal{P} 1): \min c^{T} \boldsymbol{x} \\
& \text { s.t. } \quad A_{1} \boldsymbol{x} \geq b_{1} \\
& \boldsymbol{x} \geq 0 \\
& \boldsymbol{x} \in \mathbb{Z}^{n_{1}}
\end{aligned}
$$$$
(\mathcal{P} 2): \min f^{T} \boldsymbol{y}
$$

$$
\text { s.t. } \begin{aligned}
A_{2} \boldsymbol{y} & \geq b_{2} \\
\boldsymbol{y} & \geq 0 \\
\boldsymbol{y} & \in \mathbb{Z}^{n_{2}}
\end{aligned}
$$


Methodology (1)

$$
\begin{aligned}
(\mathcal{P} 3): \min f^{T} \boldsymbol{y}, & \\
\text { s.t. } \quad A_{1} \boldsymbol{x} & \geq b_{1} \\
A_{2} \boldsymbol{y} & \geq b_{2} \\
D \boldsymbol{x} & =W \boldsymbol{y} \\
\boldsymbol{x}, \boldsymbol{y} & \geq 0 \\
\boldsymbol{x} & \in \mathbb{Z}^{n_{1}} \\
\boldsymbol{y} & \in \mathbb{Z}^{n_{2}}
\end{aligned}
$$


Methodology (1)

$$
\begin{aligned}
(\mathcal{P} 3): \min f^{T} \boldsymbol{y}, & \\
\text { s.t. } \quad A_{1} \boldsymbol{x} & \geq b_{1} \\
A_{2} \boldsymbol{y} & \geq b_{2} \\
D \boldsymbol{x} & =W \boldsymbol{y} \\
\boldsymbol{x}, \boldsymbol{y} & \geq 0 \\
\boldsymbol{x} & \in \mathbb{Z}^{n_{1}} \\
\boldsymbol{y} & \in \mathbb{Z}^{n_{2}}
\end{aligned}
$$




\section{Methodology (2)}

\section{Branch-and-Benders-Cut}

- Benders Decomposition is viewed as a Branch-and-Cut Algorithm

$$
\begin{aligned}
(\mathcal{M P}): \min & f^{T} \boldsymbol{y} \\
\text { s.t. } \quad A_{2} \boldsymbol{y} & \geq b_{2} \\
\boldsymbol{y} & \geq 0 \\
\boldsymbol{y} & \in \mathbb{R}^{n_{2}}
\end{aligned}
$$

$$
\begin{aligned}
(\mathcal{S P}): \min & 0^{T} \boldsymbol{x} \\
\text { s.t. } \quad A_{1} \boldsymbol{x} & \geq b_{1} \\
D \boldsymbol{x} & =W \bar{y} \\
\boldsymbol{x} & \geq 0 \\
\boldsymbol{x} & \in \mathbb{R}^{n_{1}}
\end{aligned}
$$

- Feasibility cuts are generated at each node of the Branch-and-Bound tree

$$
u_{1}^{T} b_{1}+u_{2}^{T} W \boldsymbol{y} \leq 0
$$

- Branching can be implemented in either problem

- Branching on the $\boldsymbol{x}$ variables could be appealing

$\rightarrow$ Implications on $(\mathcal{M P})$ enforced through feasibility cuts 


\section{Application One: Bin Packing Problem}

\section{Reformulation}

- The Cutting Stock Problem is a well known problem in the OR literature

- Involves determining the minimum number of rolls (or stocks) of a given length that must be cut in order to meet the demand for a set of items, of shorter, specified lengths.

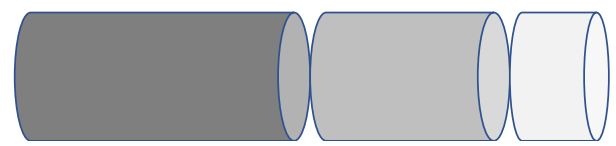

- The Bin Packing Problem is a special case of this where the cost of using a given stock is equal to one.

- Various formulations exist

- Simultaneously consider an arc-flow formulation and a path based formulation 


\section{Formulations}

The network - Carvalho (1999)

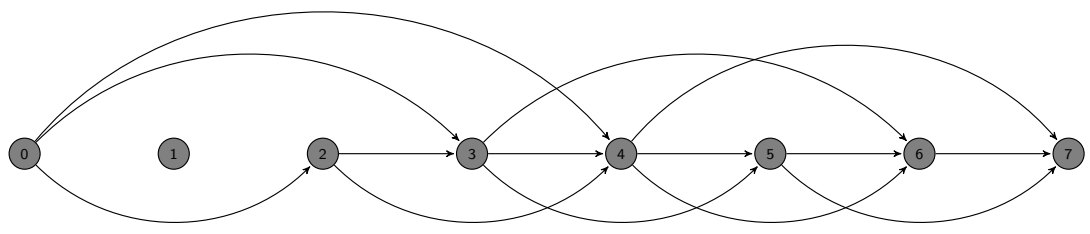

Arc flow formulation

$$
\begin{aligned}
(\mathcal{B P} 1) \min z & \\
\sum_{(j, i) \in A} \boldsymbol{x}_{j, i}-\sum_{(i, j) \in A} \boldsymbol{x}_{i, j} & = \begin{cases}z & j=0 \\
0 & j=1, \ldots, W-1 \quad \forall j \in N \\
-z & j=W\end{cases} \\
\sum_{\left(h, h+w_{i}\right) \in A} \boldsymbol{x}_{h, h+w_{i}} & \geq b_{i} \quad \forall i \in I \\
\boldsymbol{x}_{i j} & \in \mathbb{Z}^{+} \quad \forall(i, j) \in A \\
z & \in \mathbb{Z}^{+}
\end{aligned}
$$




\section{Formulations}

The network - Carvalho (1999)

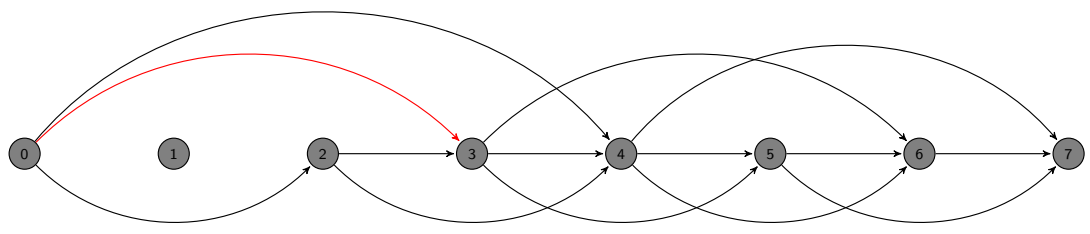

Arc flow formulation

$$
\begin{aligned}
(\mathcal{B P} 1) \min z & \\
\sum_{(j, i) \in A} \boldsymbol{x}_{j, i}-\sum_{(i, j) \in A} \boldsymbol{x}_{i, j} & = \begin{cases}z & j=0 \\
0 & j=1, \ldots, W-1 \quad \forall j \in N \\
-z & j=W\end{cases} \\
\sum_{\left(h, h+w_{i}\right) \in A} \boldsymbol{x}_{h, h+w_{i}} & \geq b_{i} \quad \forall i \in I \\
\boldsymbol{x}_{i j} & \in \mathbb{Z}^{+} \quad \forall(i, j) \in A \\
z & \in \mathbb{Z}^{+}
\end{aligned}
$$




\section{Formulations}

The network - Carvalho (1999)

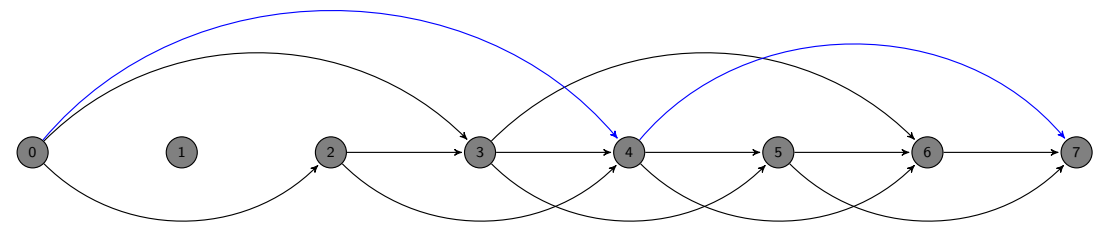

Pattern based

$$
\begin{aligned}
&(\mathcal{B P} 2) \min \sum_{p \in P} \boldsymbol{\lambda}_{p} \\
& \sum_{p \in P} a_{i p} \boldsymbol{\lambda}_{p} \geq b_{i} \forall i \in I \\
& \boldsymbol{\lambda}_{p} \in \mathbb{Z}^{+} \quad \forall p \in P
\end{aligned}
$$




\section{Methodology}

- Combine both formulations and augment with a linking constraint

$$
\begin{aligned}
& (\mathcal{B P} 3) \text { minimize } \sum_{p \in P} \boldsymbol{\lambda}_{p}, \\
& \text { s.t. } \quad \sum_{p \in P} a_{i p} \boldsymbol{\lambda}_{p} \geq b_{i} \\
& \sum_{p \in P} a_{i p} \boldsymbol{\lambda}_{p}=\sum_{\left(h, h+w_{i}\right) \in A} \boldsymbol{x}_{h, h+w_{i}} \\
& \sum_{(j, i) \in A} \boldsymbol{x}_{j i}-\sum_{(i, j) \in A} \boldsymbol{x}_{i, j}= \begin{cases}\sum_{p \in P} \boldsymbol{\lambda}_{p} & j=0 \\
0 & j=1, \ldots, W-1 \\
-\sum_{p \in P} \boldsymbol{\lambda}_{p} & j=W\end{cases} \\
& \boldsymbol{x}_{i j} \in \mathbb{Z}^{+} \\
& \boldsymbol{\lambda}_{p} \in \mathbb{Z}^{+}
\end{aligned}
$$

- Solve using Benders Branch-and-Cut

- Compare with the Branch-Price-and-Cut algorithm of Alves \& Carvalho (2008) 


\section{Methodology}

- Combine both formulations and augment with a linking constraint

$$
\begin{aligned}
& (\mathcal{B P} 3) \text { minimize } \sum_{p \in P} \boldsymbol{\lambda}_{p}, \\
& \text { s.t. } \quad \sum_{p \in P} a_{i p} \boldsymbol{\lambda}_{p} \geq b_{i} \\
& \sum_{p \in P} a_{i p} \boldsymbol{\lambda}_{p}=\sum_{\left(h, h+w_{i}\right) \in A} \boldsymbol{x}_{h, h+w_{i}} \\
& \sum_{(j, i) \in A} \boldsymbol{x}_{j i}-\sum_{(i, j) \in A} \boldsymbol{x}_{i, j}= \begin{cases}\sum_{p \in P} \boldsymbol{\lambda}_{p} & j=0 \\
0 & j=1, \ldots, W-1 \\
-\sum_{p \in P} \boldsymbol{\lambda}_{p} & j=W\end{cases} \\
& \boldsymbol{x}_{i j} \in \mathbb{Z}^{+} \\
& \boldsymbol{\lambda}_{p} \in \mathbb{Z}^{+}
\end{aligned}
$$

- Solve using Benders Branch-and-Cut

- Compare with the Branch-Price-and-Cut algorithm of Alves \& Carvalho (2008) 


\section{Methodology}

- Combine both formulations and augment with a linking constraint

$$
\begin{aligned}
& (\mathcal{B P} 3) \text { minimize } \sum_{p \in P} \boldsymbol{\lambda}_{p}, \\
& \text { s.t. } \quad \sum_{p \in P} a_{i p} \boldsymbol{\lambda}_{p} \geq b_{i} \\
& \sum_{p \in P} a_{i p} \boldsymbol{\lambda}_{p}=\sum_{\left(h, h+w_{i}\right) \in A} \boldsymbol{x}_{h, h+w_{i}} \\
& \sum_{(j, i) \in A} \boldsymbol{x}_{j i}-\sum_{(i, j) \in A} \boldsymbol{x}_{i, j}= \begin{cases}\sum_{p \in P} \boldsymbol{\lambda}_{p} & j=0 \\
0 & j=1, \ldots, W-1 \\
-\sum_{p \in P} \boldsymbol{\lambda}_{p} & j=W\end{cases} \\
& x_{i j} \in \mathbb{R}^{+} \\
& \boldsymbol{\lambda}_{p} \in \mathbb{R}^{+}
\end{aligned}
$$

- Solve using Benders Branch-and-Cut

- Compare with the Branch-Price-and-Cut algorithm of Alves \& Carvalho (2008) 


\section{Instances}

\begin{tabular}{crrr}
\hline Category $^{1}$ & $|I|$ & $W$ & Instances \\
\hline bpp1 & 120 & 150 & 8 \\
bpp2 & 250 & 150 & 14 \\
bpp3 & 500 & 150 & 11 \\
bpp4 & 1000 & 150 & 11 \\
bpp5 & 60 & 100 & 7 \\
bpp6 & 120 & 100 & 20 \\
bpp7 & 249 & 100 & 20 \\
bpp8 & 501 & 100 & 20 \\
\hline Total & & & 111 \\
\hline
\end{tabular}

Algorithm coded in $\mathrm{C}++$, uses COIN-BCP, Cplex 12.6 \& Gurobi 7.5 for LPs Max run time of 3 hours, Intel Xeon $2.6 \mathrm{GHz}$ processor, Linux

\footnotetext{
${ }^{1}$ https://www.math.u-bordeaux.fr/ fvanderb/publications.html
}

9 DTU Management Engineering 
Alves \& Carvalho (2008) Benders Approach

\begin{tabular}{l|rrr|rrrr}
\hline Class & Nodes & Price $(\mathrm{s})$ & \multicolumn{1}{c}{ Solve (s) } & Nodes & Cuts & Price (s) & Solve(s) \\
\hline bpp1 & 73.00 & 0.62 & 4.04 & 24.34 & 0.00 & 0.49 & 1.60 \\
bpp2 & 60.27 & 1.00 & 6.15 & 20.07 & 0.00 & 0.54 & 1.77 \\
bpp3 & 200.50 & 7.48 & 88.56 & 48.82 & 0.00 & 0.49 & 4.56 \\
bpp4 & 399.91 & 13.10 & 143.86 & 150.00 & 0.00 & 1.91 & 13.22 \\
bpp5 & 26.00 & 1.75 & 3.08 & 29.20 & 0.00 & 0.29 & 5.44 \\
bpp6 & 111.12 & 15.63 & 41.20 & 192.90 & 1.25 & 1.26 & 50.56 \\
bpp7 & 423.60 & 187.88 & 933.07 & 1058.90 & 9.00 & 6.43 & 505.59 \\
bpp8 & 778.80 & 769.35 & 3990.16 & 3873.50 & 67.75 & 30.56 & 3313.96 \\
\hline
\end{tabular}


Alves \& Carvalho (2008) Benders Approach

\begin{tabular}{l|rrr|rrrr}
\hline Class & Nodes & Price $(\mathrm{s})$ & \multicolumn{1}{c}{ Solve (s) } & Nodes & Cuts & Price (s) & Solve(s) \\
\hline bpp1 & 73.00 & 0.62 & 4.04 & 24.34 & 0.00 & 0.49 & 1.60 \\
bpp2 & 60.27 & 1.00 & 6.15 & 20.07 & 0.00 & 0.54 & 1.77 \\
bpp3 & 200.50 & 7.48 & 88.56 & 48.82 & 0.00 & 0.49 & 4.56 \\
bpp4 & 399.91 & 13.10 & 143.86 & 150.00 & 0.00 & 1.91 & 13.22 \\
bpp5 & 26.00 & 1.75 & 3.08 & 29.20 & 0.00 & 0.29 & 5.44 \\
bpp6 & 111.12 & 15.63 & 41.20 & 192.90 & 1.25 & 1.26 & 50.56 \\
bpp7 & 423.60 & 187.88 & 933.07 & 1058.90 & 9.00 & 6.43 & 505.59 \\
bpp8 & 778.80 & 769.35 & 3990.16 & 3873.50 & 67.75 & 30.56 & 3313.96 \\
\hline
\end{tabular}


Comments

- Proposed methodology is competitive

- Benders Decomposition evaluates more nodes

- Feasibility cuts needed for some instances only 


\section{Application Two: SDVRP Relaxation}

- Variant of the classical Capacitated Vehicle Routing Problem (CVRP)

- Given a set of Customer demands, a depot, and a fleet of vehicles with a certain capacity, partition the customers into vehicle tours that start and end at the depot, satisfy the vehicle capacities, and which minimize the transportation cost.

Formulated on a Graph $G=(V, E)$

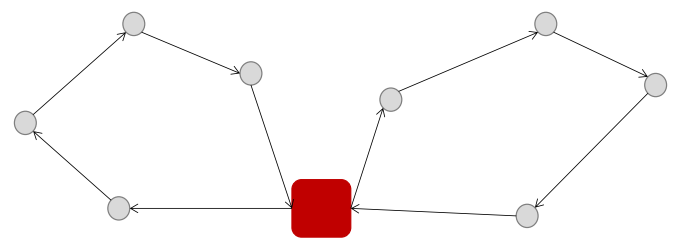

- In the SDVRP a customer can be visited by multiple vehicles

- Notoriously difficult problem

- Simultaneously consider an exact formulation and a relaxation 


\section{Formulations}

- Use the exact three index formulation of Li et al. (2006)

- $x_{i j}^{k} \in\{0,1\}$ determines with vehicle $k$ travels between nodes $i$ and $j$

- $y_{i k} \in[0,1]$ states the portion of node $i$ 's demand serviced by vehicle $k$

- $t_{i k} \geq 0$ is the time at which vehicle $k$ visits node $i$

- Combine with the two index formulation of Archetti at al. (2014)

- $x_{i j} \in\{0,1\}$ counts the number of vehicle's travelling between nodes $i$ and $j$

- $z_{i} \geq 0$ states the number of times node $i$ is visited 


\section{Formulations}

$$
\begin{aligned}
& \min \sum_{i, j \in V} \sum_{k \in K} c_{i j} x_{i j}^{k} \\
& \text { s.t. } \quad \sum_{j \in V} x_{0 j}^{k} \leq 1 \\
& \sum_{j \in V} x_{j i}^{k}-\sum_{j \in V} x_{i j}^{k}=0 \\
& t_{i k}-t_{j k}+(|V|+1) x_{i j}^{k} \leq|V| \\
& \sum_{k \in K} y_{i k}=1 \\
& \sum_{i \in I} d_{i} y_{i k} \leq Q \\
& y_{i k}-\sum_{j \in V} x_{j i}^{k} \leq 0 \\
& x_{i j}^{k} \in\{0,1\} \\
& 0 \leq y_{i k} \leq 1 \\
& t_{i k} \geq 0 \\
& \forall(i j) \in E, \forall k \in K \\
& \forall i \in V, \forall k \in K \\
& \forall i \in V, \forall k \in K
\end{aligned}
$$




\section{Formulations}

$$
\begin{array}{rrr}
\min & \sum_{i, j \in V} c_{i j} x_{i j} & \\
\text { s.t. } \quad \sum_{i \in \delta(i)} x_{i j}=2 z_{i} & \forall i \in V \\
\sum_{(i, j) \in \delta(S)} x_{i j} & \geq 2 \cdot\left\lceil\frac{\sum_{i \in S} d_{i}}{Q}\right\rceil \\
x_{i j} & \in\{0,1\} & \forall S \subset N,|S| \geq 2 \\
k_{N} \leq x_{i j} & \leq 2 \cdot|K| & \\
k_{N} & \leq z_{0} \leq|K| \text { and integer } \\
1 & \leq z_{i} \leq|K| \text { and integer } & \forall(i, j) \in E(N)
\end{array}
$$

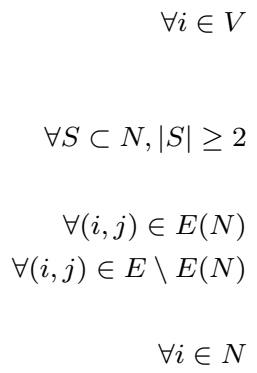




\section{Methodology}

- Couple both formulations together \& augment the resulting model with

$$
x_{i j}=\sum_{k \in K} x_{i j}^{k} \quad \forall(i, j) \in E
$$

- Apply Branch-and-Benders-Cut

- Use the two index formulation as the master problem

- Branch in the master problem

- Separate feasibility cuts on finding integer solutions

- Compare with the Branch-and-Cut Algorithm of Archetti et al. (2014)

- Dynamically, and heuristically, separate capacity cuts, connectivity cuts 


\section{Results: Proposed Methodology}

Tested on 25 instances from Belenguer et al. (2000) (Integer distances)

\begin{tabular}{lrrrrccrr}
\hline Instance & Ben. & \multicolumn{1}{c}{ Cap. } & Con. & Subs & $L P$ & $I P$ & Nodes & Time (s) \\
\hline eil22 & 0 & 47 & 0 & 1 & 375.00 & 375.00 & 1 & 0.11 \\
eil23 & 0 & 20 & 1 & 1 & 569.00 & 569.00 & 1 & 0.06 \\
eil30 & 1,216 & 3,055 & 1,430 & 2,021 & 508.00 & 510.00 & 4,679 & 109.71 \\
eil33 & 0 & 163 & 0 & 1 & 833.50 & 835.00 & 3 & 1.46 \\
eil51 & 0 & 5,221 & 81 & 12 & 514.10 & 521.00 & 237 & 17.35 \\
S51D1 & 0 & 3,59 & 0 & 20 & 454.33 & 458.00 & 73 & 9.13 \\
S76D1 & 0 & 10,750 & 80 & 16 & 586.16 & 592.00 & 399 & 63.66 \\
\hline
\end{tabular}

Algorithm coded in C ++ , uses COIN-BCP, Cplex 12.6 \& Gurobi 7.5 for LPs Max run time of 3 hours, Intel Xeon $2.6 \mathrm{GHz}$ processor, Linux 
Results: Proposed Methodology

\begin{tabular}{l|rrrrrrrrr}
\hline Instance & Ben. & \multicolumn{1}{c}{ Cap. } & \multicolumn{1}{c}{ Con. } & \multicolumn{1}{c}{ Subs } & \multicolumn{1}{c}{$L P^{1}$} & \multicolumn{1}{c}{$L P^{2}$} & \multicolumn{1}{c}{$I P$} & Nodes \\
\hline S51D2 & 533 & 466,626 & 9,441 & 3,399 & 678.21 & 687.07 & 728.00 & 20,085 \\
S51D3 & 560 & 302,259 & 5,566 & 2,761 & 905.78 & 914.31 & N/A & 14,079 \\
S51D4 & 452 & 496,903 & 4,778 & 2,094 & $1,519.78$ & $1,524.48$ & N/A & 13,089 \\
S51D5 & 560 & 539,575 & 6,118 & 2,601 & $1,279.09$ & $1,287.24$ & N/A & 17,118 \\
S51D6 & 367 & 575,843 & 369 & 1,815 & $2,126.45$ & $2,128.48$ & N/A & 9,233 \\
\hline eilA76 & 399 & 318,952 & 9,790 & 2,519 & 778.10 & 785.04 & N/A & 14,432 \\
eilB76 & 321 & 461,015 & 11,001 & 1,642 & 933.97 & 940.62 & N/A & 13,697 \\
eilC76 & 162 & $1,467,051$ & 32,989 & 1,236 & 703.78 & 709.56 & 780.00 & 39,742 \\
eilD76 & 97 & $1,373,146$ & 38,055 & 993 & 657.68 & 662.29 & 707.00 & 44,659 \\
\hline eilA101 & 24 & 914,453 & 17,926 & 298 & 788.19 & 793.13 & 872.00 & 22,541 \\
eilB101 & 153 & 299,122 & 7,956 & 742 & $1,000.99$ & $1,005.22$ & N/A & 7,119 \\
\hline S76D2 & 320 & 480,082 & 10,431 & 1,627 & $1,016.56$ & $1,022.03$ & N/A & 12,525 \\
S76D3 & 225 & 413,936 & 7,888 & 1,044 & $1,347.90$ & $1,352.61$ & N/A & 9,548 \\
S76D4 & 124 & 340,259 & 3,774 & 743 & $2,005.18$ & $2,007.16$ & N/A & 7087 \\
\hline S101D1 & 58 & 867,619 & 8,785 & 961 & 702.77 & 708.46 & 726.00 & 24,211 \\
S101D2 & 87 & 316,617 & 6,454 & 434 & $1,270.25$ & $1,276.42$ & N/A & 4,893 \\
S101D3 & 77 & 369,606 & 4,668 & 348 & $1,753.37$ & $1,755.25$ & N/A & 4,211 \\
S101D5 & 35 & 334,711 & 3,078 & 149 & $2,652.75$ & $2,652.78$ & N/A & 3,040 \\
\hline
\end{tabular}


Results: Proposed Methodology

\begin{tabular}{l|rrrrrrrrr}
\hline Instance & Ben. & \multicolumn{1}{c}{ Cap. } & \multicolumn{1}{c}{ Con. } & \multicolumn{1}{c}{ Subs } & \multicolumn{1}{c}{$L P^{1}$} & \multicolumn{1}{c}{$L P^{2}$} & \multicolumn{1}{c}{$I P$} & Nodes \\
\hline S51D2 & 533 & 466,626 & 9,441 & 3,399 & 678.21 & 687.07 & 728.00 & 20,085 \\
S51D3 & 560 & 302,259 & 5,566 & 2,761 & 905.78 & 914.31 & N/A & 14,079 \\
S51D4 & 452 & 496,903 & 4,778 & 2,094 & $1,519.78$ & $1,524.48$ & N/A & 13,089 \\
S51D5 & 560 & 539,575 & 6,118 & 2,601 & $1,279.09$ & $1,287.24$ & N/A & 17,118 \\
S51D6 & 367 & 575,843 & 369 & 1,815 & $2,126.45$ & $2,128.48$ & N/A & 9,233 \\
\hline eilA76 & 399 & 318,952 & 9,790 & 2,519 & 778.10 & 785.04 & N/A & 14,432 \\
eilB76 & 321 & 461,015 & 11,001 & 1,642 & 933.97 & 940.62 & N/A & 13,697 \\
eilC76 & 162 & $1,467,051$ & 32,989 & 1,236 & 703.78 & 709.56 & 780.00 & 39,742 \\
eilD76 & 97 & $1,373,146$ & 38,055 & 993 & 657.68 & 662.29 & 707.00 & 44,659 \\
\hline eilA101 & 24 & 914,453 & 17,926 & 298 & 788.19 & 793.13 & 872.00 & 22,541 \\
eilB101 & 153 & 299,122 & 7,956 & 742 & $1,000.99$ & $1,005.22$ & N/A & 7,119 \\
\hline S76D2 & 320 & 480,082 & 10,431 & 1,627 & $1,016.56$ & $1,022.03$ & N/A & 12,525 \\
S76D3 & 225 & 413,936 & 7,888 & 1,044 & $1,347.90$ & $1,352.61$ & N/A & 9,548 \\
S76D4 & 124 & 340,259 & 3,774 & 743 & $2,005.18$ & $2,007.16$ & N/A & 7087 \\
\hline S101D1 & 58 & 867,619 & 8,785 & 961 & 702.77 & 708.46 & 726.00 & 24,211 \\
S101D2 & 87 & 316,617 & 6,454 & 434 & $1,270.25$ & $1,276.42$ & N/A & 4,893 \\
S101D3 & 77 & 369,606 & 4,668 & 348 & $1,753.37$ & $1,755.25$ & N/A & 4,211 \\
S101D5 & 35 & 334,711 & 3,078 & 149 & $2,652.75$ & $2,652.78$ & N/A & 3,040 \\
\hline
\end{tabular}


Comments

- Struggles on larger instances

- Combine with an upper bound heuristic

- Feasibility cuts on fractional solutions 


\section{Summary}

- Proposed framework couples two formulations to exploit both

- Tested its performance using a reformulation and a relaxation

- Shows promise

- More development and testing need 


\section{Thanks for your attention!}

